\title{
Brilliant Coloured Monochromatic Photonic Crystals Films Generation from Poly (Styrene-Butyl Acrylate-Acrylic Acid) Latex
}

\section{1*IFIJEN, IH; ${ }^{3}$ MALIKI, M; ${ }^{1}$ OVONRAMWEN, OB; ${ }^{1}$ AIGBODION, AI; ${ }^{2}$ IKHUORIA, EU}

\author{
${ }^{*}$ Department of Research Operations, Rubber Research Institute of Nigeria, Iyanomo, Benin City, Nigeria \\ ${ }^{2}$ Department of Chemistry, University of Benin, P.M.B. 1154, Benin City, Nigeria \\ ${ }^{3}$ Departments of Chemistry, Edo University Iyamho, Edo State, Nigeria \\ *Corresponding Author Email: larylans4u@yahoo.com
}

\begin{abstract}
A one-pot synthetic procedure was successfully used to synthesize monodispersed poly (styrene-butyl acrylate-acrylic acid) (P (St-BA-AA)) colloidal spheres via emulsion polymerization. A glass transition temperature (Tg) of $108^{\circ} \mathrm{C}$ was obtained by DSC analysis. Core-shell morphology was revealed by TEM micrograph. Evaporative induced self-assembly process was used to fabricate photonic crystals from the as-synthesized P (St-BA-AA) latexes. Microscopic analyses of the fabricated photonic crystal films revealed the assembling of colloidal particles which rearranged into a fascinating compact three-dimensional periodic hexagonal structure with manifold layer arrangement. The photonic crystal films displayed mesmerizing monochromatic yellow, blue and green colours which were obtained by controlling the size of the microspheres. The prospect of deliberately obtaining photonic crystals with monochromatic colours would be very useful in the generation of photonic crystals for sensing application.
\end{abstract}

\section{DOI: https://dx.doi.org/10.4314/jasem.v23i9.9}

Copyright: Copyright (C) 2019 Ifigen et al. This is an open access article distributed under the Creative Commons Attribution License (CCL), which permits unrestricted use, distribution, and reproduction in any medium, provided the original work is properly cited.

Dates: Received: 14 August 2019; Revised: 22 September 2019; 27 September 2019

Keywords: poly (styrene-butyl-acrylate-acrylic acid); photonic crystals; ter-polymer; monochromatic

The pleasant and amazing viewing potential under diverse illuminating environs has attracted numerous attentions towards the generation of reflective-type displays. These reflective-type displays can be achieved by controlling the reflectance of each pixel, as such giving rise to a coloured image under a light (Kim et al., 2018). A scheme that is capable of selecting reflecting colours or a transmissive colour filter array is required for the implementation of colours in reflective-type displays. The generation of full-colour reflective-type displays using electrowetting (Yi et al., 2015), electrophoresis (Mukherjee et al., 2015) and interferometry (Hong et al., 2014), has been attempted by several studies. However, overcoming the insufficient colour depth, the low reflectivity and the colour loss is still very challenging. In recent times, photonic crystals (PCs) have attracted great attention as new materials for colour generation (Segal et al., 2015). The manipulation of either the refractive index or lattice constant by thermal (Dai et al., 2011, Markos, 2016), optical (Kim et al., 2018), and mechanical stimuli (Kim et al., 2018) can be carried out in order to tune the structural colours from the PCs. Previous studies have shown that a colour tunable PC is easier to obtain than PC with monochromatic colour (Ifijen et al., 2019).

*Corresponding Author Email: larylans4u@yahoo.com
This study, therefore, fabricated series of highly ordered three-dimensional monochromatic photonic colours from crystals of poly (styrenemethylmethacrylate-acrylic acid) colloidal microspheres via the vertical deposition technique.

\section{MATERIALS AND METHODS}

Materials: Styrene, Butyl acrylate (BA), Acrylic acid (AA), Acrylamide (AAm), Sodium dodecyl benzenesulfonate (SDBS), N, Nmethylenebisacrylamide (99\%) (BIS), ethylene glycol, N,N-methylenebisacrylamide (99\%) (BIS), Ammonium bicarbonate, (EG), (KPS), pure nitrogen gas, Ammonium per-sulphate (APS) and dimethoxyacetophenone (DMAP). All reagents are of analytical grade.

Synthesis of poly (styrene-butyl-acrylate acrylic acid) with variable diameters and coloured photonic crystal (PC) films fabrication: Monodispersed poly (styrenebutyl-acrylate-acrylic acid) (P(St-BA-AA)) colloidal microspheres were prepared via batch soap seeded emulsion polymerization technique as described by Ifijen et al. and Minghui et al. (Ifijen et al., 2019, Wang et al., 2016). In a typical experiment, sodium 
deodecyl benzene sulphurnate (SDBS) $(0.005 \mathrm{~g})$, acrylic acid (AA) $(0.720 \mathrm{~g})$, ammonium bicarbonate $(0.085 \mathrm{~g})$ and styrene $(\mathrm{St})(3.190 \mathrm{~g})$ were transferred into a $50 \mathrm{ml}$ flask containing distilled water $(16.5 \mathrm{~g})$. The mixture was stirred at $410 \mathrm{rpm}$ in a nitrogen environment at a temperature of $90{ }^{\circ} \mathrm{C}$ for about 30 minutes. The mixture of butyl-acrylate (BA) $(0.735 \mathrm{~g})$ monomer and APS initiator $(0.091 \mathrm{~g})$ was then transferred into the reaction flask. The reaction was stopped after $13 \mathrm{hrs}$.

Generation of coloured photonic crystal (PC) films: The coloured PCs were generated following a published procedure (Ifijen et al., 2019). Here, P(StBA-AA) crystal films were generated from the assynthesized latexes via the evaporative induced selfassembly of colloidal suspension made from P(St-BAAA) latex and water on the surface of glass slides. The crystal films were obtained by carrying out the assembly process at $60{ }^{\circ} \mathrm{C}$ for $24 \mathrm{hr}$ in a water bath. The self-assembling of the latex particles was instigated by the capillary force between the convective force and the interface of the glass substrate generated by the evaporation of solvent.

Characterization Techniques: High-resolution transmission electron microscopy (TECNAI F2G20 HRTEM) and Atomic force microscope in the tapping mode (Bruker Multimode, Germany), were used to determine the arrangement pattern of the prepared $\mathrm{P}$ (St-BA-AA) latex and the generated photonic crystal films. The functional groups'determination of the synthesized latex samples was achieved using Perk spectrum one FT-IR spectrometer.

\section{RESULTS AND DISCUSSION}

Figures 1,2 and 3 revealed the functional groups of $\mathrm{P}$ (St-BA-AA) latex. C-H out-of-plane bending vibrations in methylene and benzene groups are due to the peaks at $696 \mathrm{~cm}^{-1}$ and $757 \mathrm{~cm}^{-1}$ respectively.

The peaks at $1449 \mathrm{~cm}^{-1}, 1509 \mathrm{~cm}^{-1}$ and $1589 \mathrm{~cm}^{-1}$ are assigned to aromatic $\mathrm{C}=\mathrm{C}-\mathrm{C}$ stretching vibration (Omorogbe et al., 2019). The observed peaks at 2924 $\mathrm{cm}^{-1}$ and $3027 \mathrm{~cm}^{-1}$ are caused by methylene and aromatic $\mathrm{C}-\mathrm{H}$ groups respectively. The peaks at $1725 \mathrm{~cm}^{-1}$ and $1198 \mathrm{~cm}^{-1}$ are caused by carboxylic acid $(\mathrm{C}=\mathrm{O}$ stretch $)$ and $\mathrm{C}-\mathrm{O}$ stretching vibration (ester bond) (Ifijen et al., 2019) respectively.

Figure 4 revealed the SEM micrographs of the fabricated photonic crystal films with insets of coloured films and their multi-layer views. The results revealed an ordered hexagonal arrangement of spherical particles with manifold layers (Figure 4).

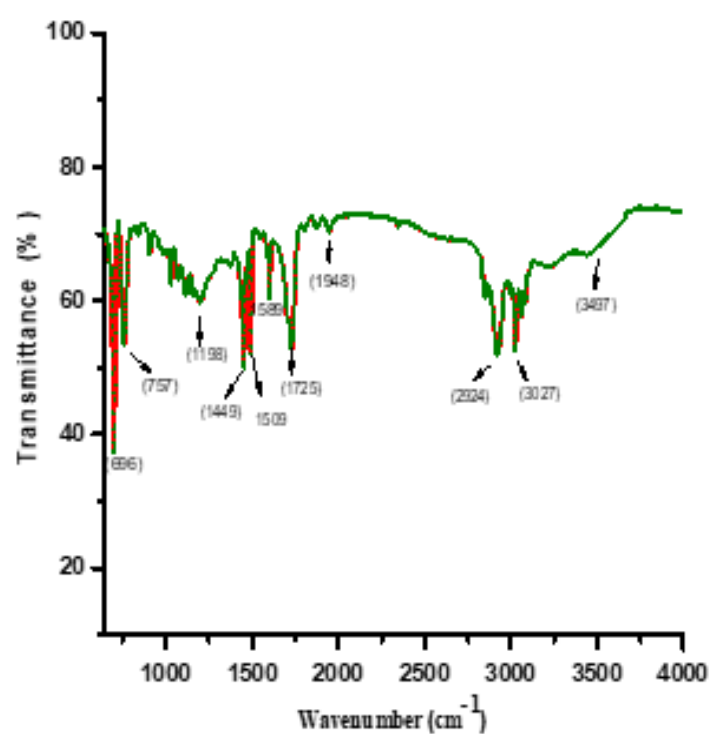

Fig. 1: Fourier transform infrared spectroscopy (FTIR) (b) Differential scanning calorimetry (c) UV-visible absorption spectra (DSC) of P(St-BA-AA) latex

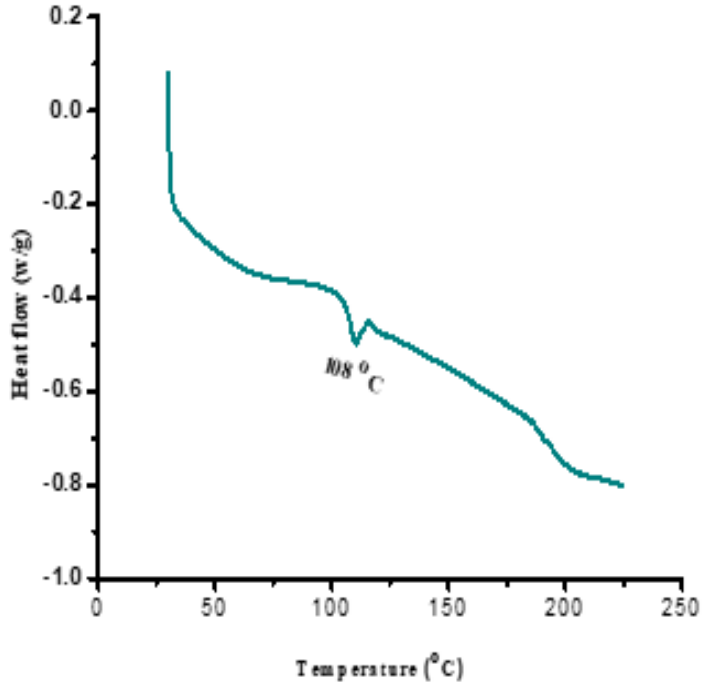

Fig 2: display the spectra of $\mathrm{P}(\mathrm{St}-\mathrm{BA}-\mathrm{AA})$ latex. A Tg of $108{ }^{\circ} \mathrm{C}$ was observed for the prepared P(St-BA-AA) latex. The obtained Tg is a pointer that the glassy and non-flexible state of the P(St-BAAA) latex can consistently be preserved whenever they are applied within the aforementioned Tg. Although, their glassy and nonflexible state becomes rubbery like when this $\mathrm{Tg}$ is passed.

The obtained average particle diameters of assynthesized $\mathrm{P}(\mathrm{St}-\mathrm{BA}-\mathrm{AA})_{1}, \mathrm{P}(\mathrm{St}-\mathrm{BA}-\mathrm{AA})_{2}$ and $\mathrm{P}(\mathrm{St}-$ BA-AA) $)_{3}$ are respectively $140 \mathrm{~nm}, 190 \mathrm{~nm}$ and 240 $\mathrm{nm}$. The films displayed brilliants yellow, blue and green colourations which did not change clour with viewing angle (monochromatic). The monochromatic nature of the obtained colours (Figure 4(a-c) can be applied in optical sensing etc. The intrinsic nature of the component materials to be used in the fabrication of responsive photonic crystals is usually considered 
when trying to generate colour responsive photonic crystals.

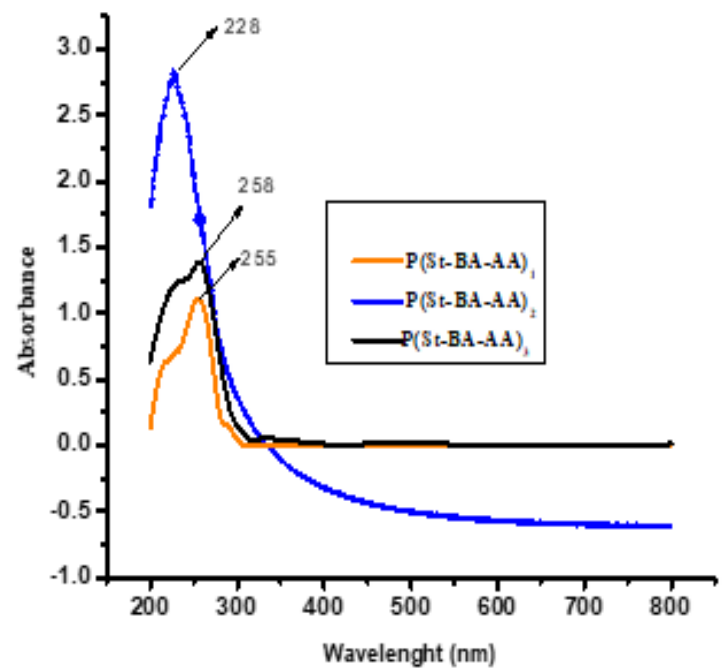

Fig 3: displays the UV spectra of $\mathrm{P}(\mathrm{St}-\mathrm{BA}-\mathrm{AA})$ latex. The result revealed a typical UV absorption for Pi-bonding to Pi-anti-bonding transformation $(\pi \rightarrow \pi *)$ at about $228 \mathrm{~nm}, 258$ and $255 \mathrm{~nm}$ respectively. This indicates the presence of conjugated double bonds latex structures.
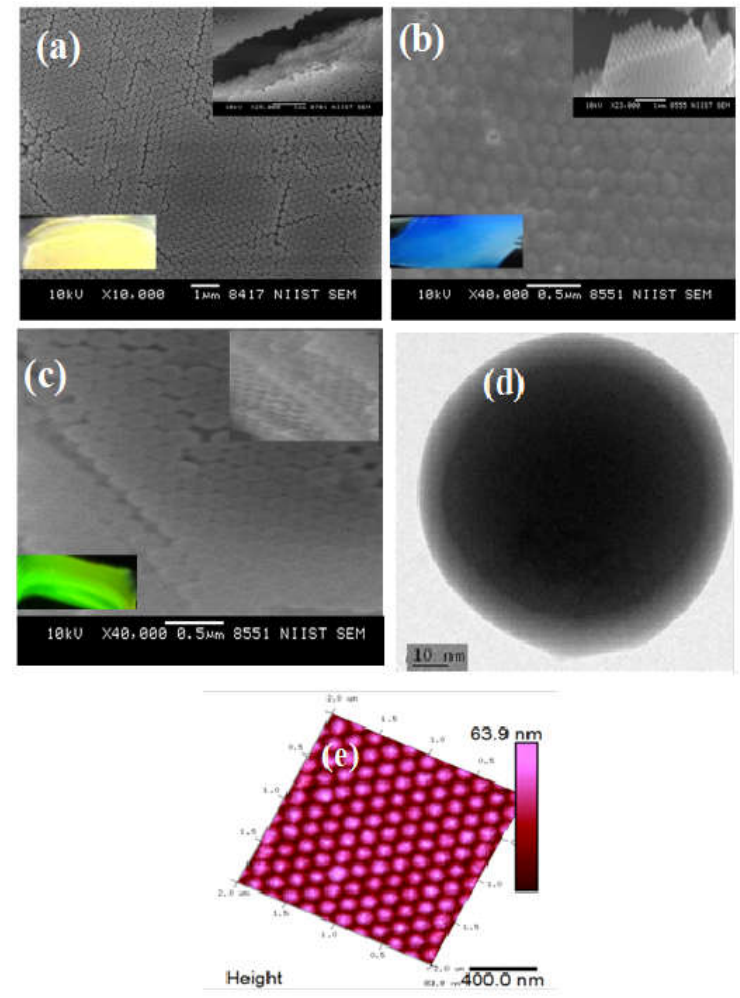

Fig 4: Scanning electron micrographs (SEM) of (a) P(St-BA-AA) (b) P(St-BA-AA $)_{2}$ (c) P(St-BA-AA $)_{3}$ with insets of multilayer view and coloured $\mathrm{PC}$ films.

For instance, in order to generate a photonic crystal film that can be responsive to a specific external stimuli with respect to colour change, the material must have an intrinsic property that is monochromatic in nature before the incorporation of a responsive molecular agent. The reason for this is because, when a monochromatic coloured material with molecular recognition agent incorporated into its matrix, changes colour when exposed to a specific external stimuli, one can be absolutely sure that, the observed change in colour is due to the external stimuli that the responsive agent is supposed to sensitive to. TEM analysis revealed two varied layers which signified core-shell morphology (Figure 4d); the dark layer (core) can be ascribed to polystyrene (PS) group, while the light layer (shell) can be attributed to butyl acrylate (BA) and acrylic acid (AA) groups. Figure 4e shows the atomic force microscopic analysis of the fabricated photonic crystal films. The micrograph revealed a three dimensional well-ordered arrangement of crystal particles which is in agreement to the obtained SEM micrograph in Figure 4a, b and c.

Conclusion: This study successfully fabricated photonic crystal films with beautiful yellow, blue and green monochromatic colours from as-synthesized poly (styrene-butylacrylate) latexes by varying their particle diameters. The possibility of deliberately obtaining photonic crystal films with monochromatic colours by controlling the sizes of the microspheres would be very useful in the creation of photonic crystals for sensing applications.

Acknowledgements: The authors appreciate the financial support of TWAS for this research and also the parents of Dr Ifijen, Ikhazuagbe Hilary (Mr. \& Mrs Ifijen) for their support.

\section{REFERENCE}

dai, X., Xiang, Y., Wen, S; He, H. (2011). Thermally tunable and omnidirectional terahertz photonic bandgap in the one-dimensional photonic crystals containing semiconductor InSb. $J$. Appl. Physics, 109, 053104.

Hong, J., Chan, E., Chang, T., Fung, R., Kim, C., Ma, J., Pan, Y., Wen, B., Reines, I; Lee, C. (2014). 54.4L: Late-News Paper: Single Mirror Interferometric Display - A New Paradigm for Reflective Display Technologies. SID Symposium Digest of Technical Papers, 45, 793-796.

Ifijen, H. I., Ikhuoria, E. U; Omorogbe, S. O. (2019). Correlative studies on the fabrication of poly(styrene-methyl-methacrylate-acrylic acid) colloidal crystal films. J. Dispersion Sci. Technol. 1-8. 
Kim, S.-U., Lee, S.-H., Lee, I.-H., Lee, B.-Y., Na, J.H; Lee, S.-D. (2018). Generation of intensitytunable structural color from helical photonic crystals for full color reflective-type display. Optics Express, 26, 13561-13572.

Markos, C. (2016). Thermo-tunable hybrid photonic crystal fiber based on solution-processed chalcogenide glass nanolayers. Scientific Reports, 6, 31711.

Mukherjee, S., Hsieh, W. L., Smith, N., Goulding, M; Heikenfeld, J. (2015). Electrokinetic pixels with biprimary inks for color displays and color-temperature-tunable smart windows. Appl Opt, 54, 5603-9.

Omorogbe, S. O., Ikhuoria, E. U., Ifijen, H. I., Simo, A., Aigbodion, A; Maaza, M. (2019).
Fabrication of Monodispersed Needle-Sized Hollow Core Polystyrene Microspheres. 2019 Cham. Springer International Publishing, 155164.

Segal, N., Keren-Zur, S., Hendler, N; Ellenbogen, T. (2015). Controlling light with metamaterialbased nonlinear photonic crystals. Nature Photonics, 9, 180.

Wang, M., Meng, F., Wu, H; Wang, J. (2016). Photonic Crystals with an Eye Pattern Similar to Peacock Tail Feathers. Crystals, 6, 99.

Yi, ZA; Shui, L; Wang, L Jin, M., Hayes, R; Zhou, G. (2015). A novel driver for active matrix electrowetting displays. $J$ Displays, 37, 86-93. 